\title{
A COMPARISON OF THE TEACHING PRACTICES OF NOVICE EDUCATORS IN ENGINEERING AND OTHER POST-SECONDARY DISCIPLINES
}

\author{
Nancy Nelson, Robert Brennan \\ Schulich School of Engineering, University of Calgary \\ nancy.nelson1@ucalgary.ca rbrennan@ucalgary.ca
}

\begin{abstract}
There is a perception in higher education that engineering educators teach differently than those in other disciplines. Surveys of student engagement consistently rank the undergraduate engineering experience lowest among ten disciplines, as do faculty surveys of student engagement. These results suggest there is opportunity and need to improve the engineering education experience.

This research sets out to identify differences in the teaching practices of beginning engineering educators from those in other disciplines. Using the Dreyfus and Dreyfus model of skill acquisition as a framework, this study examines institutional data collected during four consecutive terms of mandatory teaching observations of new full-time and selected part-time instructors.

Descriptive statistics found that the performance of novice educators in engineering-related disciplines did rank lowest overall compared to all other disciplines. This analysis also found that there is little difference in the teaching practices of novice engineering educators from those of their more experienced colleagues. Thematic analysis found that traditional engineering classroom practices such as lecture and worked examples are common, and could be enhanced by including opportunities for meaningful active learning.

These results can inform both engineering educators and those responsible for their educational development about the common teaching practices of novice instructors and will be useful in shaping the professional development opportunities offered to engineering educators.
\end{abstract}

Keywords: engineering education; teaching practices; active learning; skill acquisition; novice; educational development; professional development

\section{INTRODUCTION}

Studies show that the undergraduate engineering experience is less engaging than that in other disciplines. It is rare however, for anyone other than instructors and their students to know what happens in an undergraduate engineering classroom. How effective are these teaching practices? Is there an appropriate level of academic rigour? Is the focus on learning or teaching? Is there a strong relationship between the instructor and their students? Answers to these questions may help determine why the engineering education experience is not as engaging as it could be.

To answer these questions, one must have a first-hand view of the teaching practices of engineering educators. This paper reports the results of a mixed method analysis of mandatory observations of novice post-secondary educators. Its goal is to determine why the engineering learning experience may be less engaging that of other disciplines.

\section{BACKGROUND}

Studies show that the experience of undergraduate engineering students is less engaging than that of their peers in other disciplines. This section examines the literature on effective teaching practices, perceptions of student engagement, and the educator's journey from novice to expert.

\subsection{Effective Teaching Practices}

Research into effective educational practices identifies a number of benchmarks used to define and measure effective learning and student success. These can be categorized into six broad themes: (1) an appropriate level of academic rigour, (2) a focus on learning, (3) supported instruction, (4) quality of teaching, (5) development of strong relationships, and (6) student engagement [1][2][3].

A 2018 study shows that most Canadian undergraduate engineering classes are teacher-directed and lecture-based [4]. Some educators incorporate active and collaborative learning, but others identify barriers such as student resistance, low teaching evaluations, time commitment vs. benefit, and access to resources as reasons for their more traditional methods. In fact, Science, Technology, Math, and Engineering (STEM) educators rated the barriers to 
using active learning higher than faculty in all other disciplines [5], despite studies that indicate the efficacy and benefits of using active learning in the classroom [6][7][8][9]. Of all post-secondary educators, those in STEM most strongly believed that "active learning takes too much class time causing the coverage of content to suffer" [5].

\subsection{Perceptions of Student Engagement}

Prior work identified that engineering students around the world are less positive about their undergraduate experience than peers in other disciplines [10]. Student engagement in four areas, learning strategies, effective teaching practices, quality of interaction, and a supportive learning environment, was ranked lowest of all the disciplines (see Figure 1).

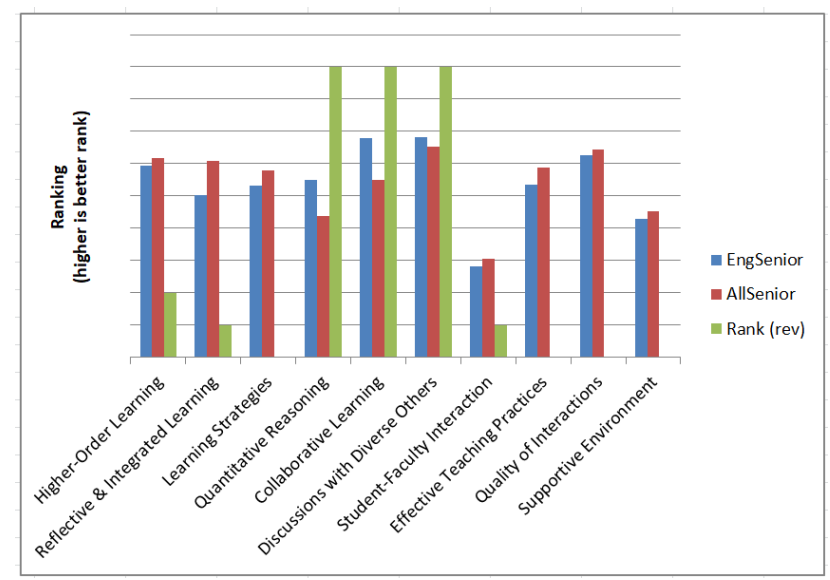

Figure 1: Ranked perception of student engagement [10]

Faculty at many post-secondary institutions around the world also participate in annual surveys looking at student engagement. The Faculty Survey of Student Engagement (FSSE) is administered to over 11,000 instructors across North America and asks questions based on the same set of benchmarks as the National Survey of Student Engagement (NSEE). An analysis of the 2018 results shows similar results to the student survey [11]; engineering ranks lowest of all disciplines in the benchmarks associated with learning strategies, discussion with diverse others, effective teaching practices, and supportive learning. Quality of interaction and reflective and integrated learning were also ranked very low compared to other disciplines (see Figure 2).

\subsection{Educator's Journey from Novice to Expert}

Shulman suggests that growth as an educator requires four types of knowledge [12]. First an instructor needs subject matter content knowledge, an understanding of the ways in which the basic concepts and principles of one's discipline are organized. This is not normally a concern for new engineering faculty members since they are hired for their technical expertise. Pedagogical knowledge of teaching, the second type, often presents a challenge for novice instructors; they do not have a solid understanding of the general principles of teaching, things like classroom organization, management, and delivery. Both content and pedagogical knowledge are required for instructors to progress to the next levels of required knowledge.

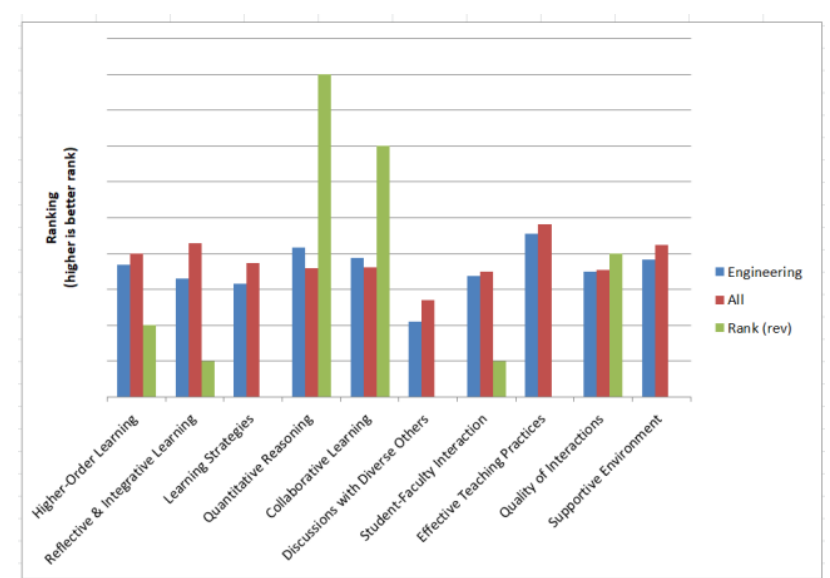

Figure 2: Faculty perception of student engagement [11]

Pedagogical content knowledge (PCK) takes subject matter content knowledge and extends it to how it should be taught. It considers the "ways of representing and formulating the subject to make it comprehensible to others" including, but not limited to, examples, analogies, illustrations, demonstrations, and explanations [12]. Educators need to recognize what makes concepts easy or difficult to learn, and to know how to help students reorganize their learning when they have misconceptions.

The final type of knowledge, related to curriculum, is an understanding of alternate ways in which specific topics can be taught at different levels of study, the variety of materials available to teach at those levels, and the reasons why one would choose whether or not to use a particular curriculum or set of materials. It also includes lateral and vertical curriculum knowledge that considers and connects with what students are learning in courses taken at the same time, previously, and in upcoming related courses.

Novice educators typically have a deep understanding of subject matter content, but may lack pedagogical, PCK, and/or curricular knowledge. As they acquire more experience, their understanding of the curriculum and ways in which to teach the content become a part of their "being" as an instructor.

Dreyfus and Dreyfus describe a five-stage model of skill acquisition where individuals progress through a series of levels from novice through competent to expert [13]. This model has been recognized as defining aspects of expertise, identifying the limitations of procedural approaches, and emphasizing the importance of tacit knowledge. It has been used to examine the journey of 
early career to seasoned professionals in varied disciplines including teaching [14][15].

Novices are generally described as individuals who clearly and objectively recognize the elements required to perform their duties. They depend on rules and procedures to make decisions, but often have difficulty predicting the outcome due to lack of experience. Educators performing at a novice level tend to rely on predetermined, structured curriculum (e.g. a textbook) and deliver information without significant context. They have content but minimal pedagogical knowledge.

Advanced beginners have accumulated some experience. They begin to recognize situations that occur in their day-to-day teaching practice, and although they often rely on the externally-provided rules and procedures, they begin to form their own set based on their own experiences, good or bad. Educators performing at an advanced beginner level need to be very organized in what and how they deliver content. They start tapping into the skills and knowledge of expert teachers, and begin to acknowledge that the learner plays a significant role in the teaching process. At this level educators are now developing pedagogical knowledge.

Competent performers are at the middle level of the novice to expert model. These individuals are able to prioritize the information and procedures necessary to make rational decisions. They are vested in the outcomes of their work and take responsibility for their own decisions. Educators performing at the competent level have a good understanding of various instructional methods and are better able to prioritize what and how information should be packaged for delivery. Assessments and outcomes become aligned and provide useful feedback to learners. Competent educators have expanded pedagogical knowledge and a sense of curricular knowledge. Unfortunately many do not move beyond this phase in the novice to expert journey since there is a "transformation, a qualitative leap, from the competent to proficient levels of performance" [14].

Proficient performers are those who can rely on their intuition. They know, understand, and can organize what they need to do. They continually take stock of a situation and adjust their methods as required. Proficient educators see the curriculum more holistically and are able to make connections between what they are teaching and the broader discipline. They recognize that they can make a difference in the lives and careers of their students, and their focus moves from teaching to learning. Instructors' curricular knowledge has expanded and they are beginning to accumulate pedagogical content knowledge.

Experts perform fluidly. They intuitively know what needs to be done and respond accordingly. They rely on their experience, no longer needing to make specific decisions related to their work. Expert educators are more spontaneous in their presentations and more intuitive in their assessment. Their methods become more experimental, recognizing that the outcome is not guaranteed. Expert educators also model what it means to be a professional in their discipline, inspiring students to become that practitioner. They are concerned about the success of their learners and shape the learning experience to support that success. Expert educators regularly apply pedagogical content knowledge in their classes.

This research sets out to identify differences in the teaching practices of novice engineering educators from those in other disciplines. Using the educator's journey from novice to expert as a framework, this study examines institutional data collected during mandatory teaching observations of new full-time faculty members and randomly selected part-time instructors. It seeks to answer the following questions: (1) is there a difference between novice educators in engineering-related disciplines and those in other disciplines? and (2) what teaching practices do novice engineering instructors demonstrate that support or impede student learning?

\section{METHODOLOGY}

This study examined institutional data collected during four semesters of mandatory teaching observations of new full-time faculty members and randomly selected parttime instructors. These developmental observations were normally performed mid-semester by either a full-time Teaching and Learning Consultant or a trained part-time retired professor. Observations were at least one hour long and may, depending on the perceived need, extend into a second hour. Prior to the observation, instructors were asked to provide digital copies of the course outline, their lesson plan, and any slides or resources to be used.

The observation instrument included 65 three-level (yes, somewhat, no) Likert scale questions that were aligned with the institutional Student Assessment of Teaching (SAT) used for performance evaluations. The 65 questions focused on six main areas: (1) lesson elements, (2) clarity and subject matter expertise (SME), (3) delivery skills, enthusiasm, and rapport, (4) teaching skills, (5) diversity, inclusion, and accessibility, and (6) legal obligations. Observers had the option of adding an explanatory comment alongside each of these questions. Observers also summarized the observed class by specifying three observed strategies that support student learning, and three opportunities to enhance practice as a professional educator. Instructors were provided with a digital copy of the observation instrument prior to the scheduled observation.

Observers completed and submitted a digital copy of the observation instrument and received a digital observation report. This report was sent to the instructor for review and reflection prior to a coaching session with the observer. The process was complete once the report and instructor reflections were sent to the instructor's department head. 


\subsection{Data Analysis}

585 developmental teaching observations were performed over four semesters starting in September 2018 and ending in December 2019. Of the 585 observations, 571 identified the school in which the instructor taught (97.6\%). Only the data from these 571 observations are included in this analysis. Table 1 shows the number and percentage of observations from each discipline.

The majority of the 571 instructors were part-time employees $(83.2 \%)$ with the remaining $16.8 \%$ being fulltime faculty members in their first semester of teaching.

Mean scores and overall rankings for each category of Likert questions are shown in Table 2. Responses were numerically coded with 'Yes' as 3, 'Somewhat' as 2, and 'No' as 1. Any 'Not Applicable' (N/A) responses were coded as 0 . Colours in the chart represent the range from lowest (reds), through average (oranges and yellows), to highest (greens).

Deductive thematic analysis was used to code the summary comments provided by the observers. 515 items were coded from the strategies that support student learning comments, and 357 from the opportunities to enhance practice responses.

Initial coding analysis produced 31 open codes for the observer comments (e.g. delivery, feedback, materials, active learning, etc.). These 31 open codes were then categorized to align with the benchmarks used in the national student engagement surveys: collaborative learning, effective teaching practices, learning strategies, and quality of instruction.

\section{RESULTS}

This section reports on the general performance, strengths and opportunities for growth of the engineering instructors who were observed early in their academic careers. It reflects aspects of their practice as compared to benchmarks used in institutional student evaluations of teaching, and those used in national student engagement surveys.

Table 1: Number of Observations by Discipline

\begin{tabular}{|l|c|c|}
\hline Discipline & $\begin{array}{c}\text { Number of } \\
\text { Observations } \\
\text { (PT/FT) }\end{array}$ & $\begin{array}{c}\text { Percentage } \\
\text { of } \\
\text { Observations }\end{array}$ \\
\hline Humanities & $45(44 / 1)$ & $7.9 \%$ \\
\hline Biological Science & 0 & $0.0 \%$ \\
\hline Physical Science & 0 & $0.0 \%$ \\
\hline Social Science & 0 & $0.0 \%$ \\
\hline Business & $194(165 / 28)$ & $34.0 \%$ \\
\hline $\begin{array}{l}\text { Communications, } \\
\text { Media \& Public } \\
\text { Relations (CM\&PR) }\end{array}$ & $103(85 / 11)$ & $18.0 \%$ \\
\hline Education & 0 & $0.0 \%$ \\
\hline Engineering & $104(79 / 21)$ & $18.2 \%$ \\
\hline Health & $28(20 / 7)$ & $4.9 \%$ \\
\hline Social Services & $29(22 / 7)$ & $5.1 \%$ \\
\hline Other & $68(49 / 19)$ & $11.9 \%$ \\
\hline count: & $571(464 / 94)$ & \\
\hline
\end{tabular}

\subsection{Descriptive Analysis}

The teaching practices of novice instructors in engineering-related disciplines are observed to be less effective than novice instructors in all other disciplines (see Figure 3). Their mean scores $(M=3.68, S D=0.17)$ were lower than all of their peers in three of the six categories measured (lesson elements, delivery, and teaching). The remaining scores (clarity, the ability to meet the needs of a diverse classroom, and meeting legal obligations) were ranked second or third lowest (see Figure 4).

Table 2: Mean Scores (and Rank) for Likert Responses

\begin{tabular}{lcccccccc} 
& $\begin{array}{c}\text { Lesson } \\
\text { Elements }\end{array}$ & $\begin{array}{c}\text { Clarity } \\
\text { SME }\end{array}$ & Delivery & Teaching & Diversity & Legal & Overall \\
\hline Humanities & $3.67(4)$ & $3.84(5)$ & $3.72(5)$ & $3.63(5)$ & $3.79(5)$ & $3.84(7)$ & $3.75(5)$ \\
Business & $3.57(6)$ & $3.79(7)$ & $3.66(6)$ & $3.58(6)$ & $3.77(7)$ & $3.89(6)$ & $3.71(6)$ \\
CM\&PR & $3.73(2)$ & $3.86(4)$ & $3.81(3)$ & $3.72(3)$ & $3.88(3)$ & $3.91(4)$ & $3.82(3)$ \\
Engineering & $3.45(7)$ & $3.82(6)$ & $3.62(7)$ & $3.53(7)$ & $3.78(6)$ & $3.89(5)$ & $3.68(7)$ \\
Health & $3.76(1)$ & $3.94(1)$ & $3.85(1)$ & $3.77(1)$ & $3.92(2)$ & $3.98(1)$ & $3.87(1)$ \\
Social Services & $3.65(5)$ & $3.87(3)$ & $3.82(2)$ & $3.75(2)$ & $3.93(1)$ & $3.95(2)$ & $3.83(2)$ \\
Other & $3.67(3)$ & $3.92(2)$ & $3.76(4)$ & $3.66(4)$ & $3.86(4)$ & $3.93(3)$ & $3.80(4)$ \\
& Mean & 3.64 & 3.86 & 3.75 & 3.66 & 3.85 & 3.91 & 3.78
\end{tabular}




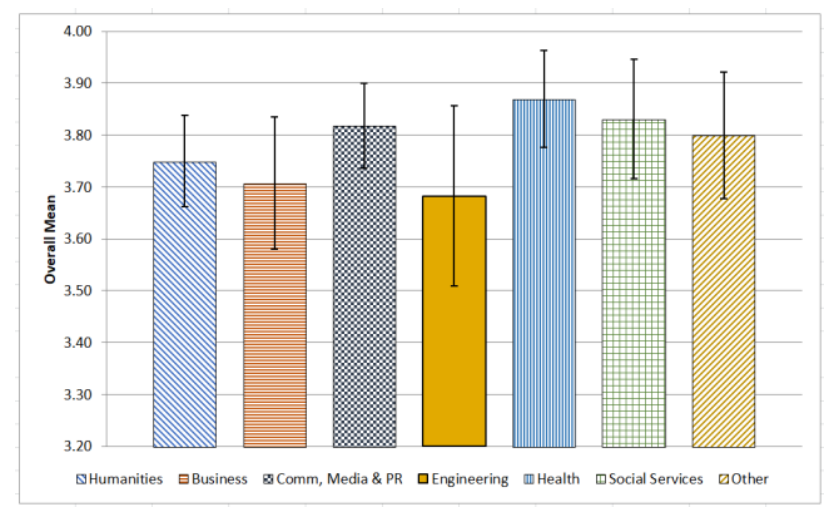

Figure 3: Discipline mean scores across all categories

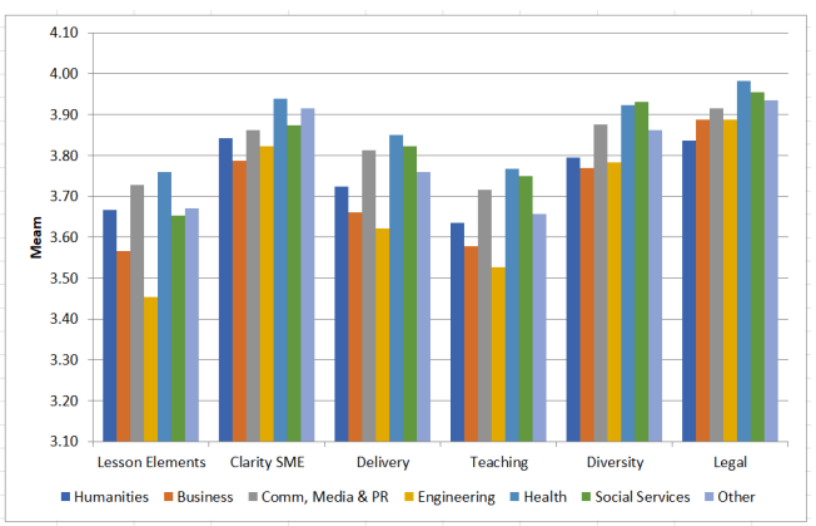

Figure 4: Discipline mean scores by category

Kruskal-Wallis $\mathrm{H}$ tests showed that there were statistically significant differences in lesson element scores between the different schools, $\chi 2(6)=41.352, p<$ 0.005 , in delivery scores between the different schools, $\chi 2(6)=10.081, p<0.005$, in teaching scores between the different schools, $\chi 2(6)=30.843, p<0.005$, and in diversity scores between the different schools, $\chi 2(6)=$ $22.083, p<0.005$. There was no statistically significant difference between schools/disciplines in the observations related to clarity/subject matter expertise or adherence to human rights, accessibility or copyright regulations.

\subsection{Thematic Analysis}

Of the 872 coded items $67.5 \%$ related to effective teaching practices. Another $25.6 \%$ related to the quality of interactions, $6.5 \%$ to collaborative learning, and the remaining $0.3 \%$ to learning strategies. There were no coded items associated with any of the other six benchmarks used in the national student engagement surveys.

The most frequently coded item related to processes associated with the teaching practices that help students co-creating knowledge (11.5\%). This encompassed items associated with ways in which problem solving is modeled, scaffolded and practiced. $78.0 \%$ of these coded items were reported as strategies that support learning and included comments such as "Effectively models and clearly explains the steps in the problem-solving process", "You shone at discussing and providing concrete examples for learners", and "You share relevant and interesting examples from your own professional experiences, and students are highly interested in these". The remaining $22.0 \%$ of the coded items were opportunities for growth. Comments included "Incorporate in-class practice time where students can actively and meaningfully engage with the new concepts", "Let them solve problems on their own and then walk them through the process. Make the class more about learning and less about teaching", and "Vary the lecture technique by introducing practice examples".

The second and third most frequently coded items related to the quality of interactions between instructors and their students. $9.5 \%$ of the items were associated with the way classrooms were managed. $72.3 \%$ of these management-related items were reported as strategies that support student learning comments. Comments included "You are employing classroom management techniques to foster a quiet, respectful classroom while treating your students respectfully as adults" and "well-managed class where students appeared to be comfortable and willing to contribute". The remaining $27.7 \%$ were presented as opportunities for growth and included comments like "You frequently interrupted and spoke over the students" and "There was some significant level of distraction which could have been impacting not only on your delivery but also on other students trying to learn".

$7.7 \%$ of the coded responses related to the instructors' personal characteristics such as enthusiasm, passion, rapport, patience, approachability, and caring and included comments like "You have a warm and approachable manner which students should find welcoming" and "Your invitational and professional teaching style encourages student engagement, creates a positive learning environment and motivates students to do their best". All coded items in this area were reported as strengths.

The three most coded items in the strategies that support student learning comments were processes associated with the teaching practice that help students co-create knowledge $(8.9 \%)$, items related to the instructors' personal characteristics $(7.7 \%)$, and the instructors' subject-matter expertise (7.0\%). Comments associated with this area included "it is obvious that you bring a wealth of experience and knowledge to this course, both in terms of study and practical techniques" and "You are an experienced subject matter expert with the ability to explain concepts".

The most commonly coded items in the opportunities to enhance practice responses were classroom management $(6.9 \%)$, in-class questioning techniques $(3.8 \%)$, and class delivery style (1.8\%). Comments relating to questioning included "Adjust your questioning technique so they invite the class to develop/use their 
higher-order thinking skills" and "Engage in more intentional lines of questioning and know what your question is for - analysis, comprehension, application, gauging understanding, etc.". Class delivery comments suggested improvement related to "you were "telling" about the content rather than teaching it" and "You need to focus on helping the students learn the material through a deliberate set of inputs, practices, feedback".

\section{DISCUSSION}

This study measured the performance of novice instructors in engineering-related classrooms compared to those in other disciplines. It also examined the comments provided to these instructors identifying strategies they are currently using that support student learning, and opportunities for enhancing their teaching practice.

It offered a rarified view into the classroom as viewed by educational developers and experienced educators. The data was explored using the instructors' novice to expert journey as measured through the benchmarks of effective education practices. It highlights three key findings:

1. the teaching practices of novice engineering educators are less effective than those of their colleagues in other disciplines, which could be addressed by tweaking the engineering culture

2. there is little difference in the teaching practices of novice engineering educators from those of their more experienced colleagues, which could be addressed through discipline-specific educational development

3. traditional engineering classroom practices such as lecture and worked examples could be enhanced by including opportunities for meaningful active learning

It is understandable that the teaching practices of novice educators would be less effective than those of their more experienced colleagues, but it seems that engineering educators begin their careers less equipped to teach than their novice colleagues in other disciplines. Each instructor in this study was required to attend a series of five mandatory teaching workshops prior to or during their first teaching semester. These workshops focused on teaching requirements and practices, assessment, creating an effective learning environment, services to support student and faculty success, and supporting student learning using the institution's learning management system.

Darling-Hammond suggests that there are three problems in learning how to teach: (1) apprenticeship of observation, (2) enactment, and (3) complexity [16]. The apprenticeship of observation suggests that people enter the teaching profession believing that teaching is the transfer of information. Unfortunately this is not aligned with what is now known about how students learn [17]. The problem of enactment identifies the challenges associated with preparing and delivering effective classes. This includes but is not limited to: organizing and presenting concepts clearly, asking and responding to relevant questions, creating effective resources and assessments, and managing the classroom. The problem of complexity challenges educators to manage and balance the needs of the students themselves, and the curriculum. Knowing which topics should be emphasized, helping students develop a deep understanding of the content, and dealing with challenging students are all examples of the complexity educators deal with every day.

The types of challenges identified during the novice instructors' observations may be explained by the three problems associated with learning to teach, but those problems do not explain why novice engineering educators are less effective than their peers in other disciplines. Nor does it explain why novice engineering instructors are perceived to be as effective as their more experienced colleagues within the discipline.

The existing culture of engineering may provide a viable explanation for these differences. A recent study identified nine themes that describe that culture [18]. The first six themes describe characteristics that are present in the culture: (1) problem-solving, (2) safety-consciousness, (3) pride in the profession, (4) loyalty, (5) flexibility in working practices, and (6) an appreciation of teamwork. Each of these characteristics is important in engineering practice, but not necessarily in establishing an effective engineering classroom. The remaining three themes describe tensions in the engineering culture and may shed insights into why engineering educators appear to be less effective than their colleagues in other disciplines.

The first tension identifies that the engineering culture is a "friendly but not personal atmosphere". Engineers tend to keep to themselves and focus on their work, not on people. Prior work showed that engineering instructors have a significant impact on student learning [10]. Other studies found that, in general, respected professors make themselves accessible to their students, demonstrate presence and caring, promote learning, keep their teaching practices current, and are enthusiastic and passionate about teaching [19] [20]. It may therefore be that an engineer's impersonal and objective nature affects classroom interactions, indirectly causing the difference between the disciplines.

The second tension identified in engineering cultures is a strong attachment to tradition. Engineers consider themselves to be problem solvers and not necessarily innovators, recognizing that they are slow to change culture [18]. Both this study and the literature show that lecturing is "deeply embedded in the culture of teaching 
in higher education" even though research suggests that a more active classroom improves learning [21][22][6]. This prevalence of traditional delivery of engineering content knowledge seems to indicate a reluctance or unawareness of curricular and/or pedagogical content knowledge. This too may contribute to the difference between the disciplines.

The third and final tension identified in engineering cultures is a lack of support and clarity in relation to career development. Engineers want more opportunities to progress as practicing engineers without going into management roles. In engineering schools this might translate into a desire to further their discipline-specific research studies. Since teaching is not their primary focus, these educators may make little progress along the novice to expert teaching journey without direction and encouragement from their deans, and opportunities to participate in level-appropriate, discipline-specific educational development opportunities.

There is a possibility that the findings of this survey have a bias associated with observation. Those observers who were familiar with the subject-matter content may have reported different views from those who did not. This may limit the generality of the results of this study. It is also not known what other factors may have contributed to the observers' responses. As a result, the following findings should be considered in the context of the limitations of this study.

\section{CONCLUSIONS AND FUTURE WORK}

This research supports and extends the findings of myriad studies that report the educational experience of undergraduate engineering students to be less effective than those of their peers in other disciplines. This study adds the insight that novice instructors in engineeringrelated disciplines demonstrate the same teaching practices as their more experienced colleagues even though they have little to no pedagogical knowledge. This suggests a reluctance or challenge on the part of engineering educators as a whole, to move beyond competent teaching by exploring and expanding their pedagogical content knowledge. Engineering culture suggests that the learning experience of undergraduate engineering students may only align with that of their peers when their instructors tap into discipline-specific, level-appropriate educational development opportunities.

This research lays the foundation for further study. Work could be done to examine the effect that disciplinespecific educational development has on teaching practices by continuing to monitor student, faculty and observer perceptions and rankings. These educational development opportunities could encourage programs to embrace the practices of proficient and expert educators by exploring and applying discipline-specific pedagogical content knowledge.

Additional research could be done to determine which levels of pedagogical, curricular, and pedagogical content knowledge engineering educators actually possess. This work could be used to shape the educational development opportunities offered to engineering instructors at various stages of their novice to expert journey as educators.

It is hoped that adding the findings of this research to the existing body of evidence on engineering educators' teaching practices will encourage engineering educators, and those responsible for their educational development, to recognize the need for discipline-specific, levelappropriate opportunities to develop their pedagogical, curricular, and pedagogical content knowledge.

\section{Acknowledgements}

This research study is funded by the National Sciences and Engineering Research Council of Canada (NSERC) and approved by both the University of Calgary Conjoint Faculties Ethics Board and the Conestoga College Research Ethics Board. Special thanks to the peer reviewers for their time and invaluable feedback.

\section{References}

[1] G. D. Kuh, J. Kinzie, J. H. Schuh, and E. Whitt, Student success in college: Creating conditions that matter. San Francisco, CA: Jossey-Bass, 2005.

[2] V. Tinto, "Enhancing student success: Taking the classroom success seriously," Int. J. First Year High. Educ., vol. 3, no. 1, pp. 1-8, 2012.

[3] National Survey of Student Engagement, "Engagement Insights: Survey Findings on the Quality of Undergraduate Education - Annual Results 2018," 2018.

[4] N. Nelson and R. Brennan, "Snapshot of engineering education in Canada," CEEA Conf. Proc. 2018, pp. 110, 2018.

[5] J. Allen, "Faculty Approaches to Active Learning: Barriers , Affordances and Adoption," Georgia State University, 2018.

[6] S. Freeman et al., "Active learning increases student performance in science, engineering, and mathematics," Proc. Natl. Acad. Sci., vol. 111, no. 23, pp. 8410-8415, 2014.

[7] D. C. Haak, J. HilleRisLambers, E. Pitre, and S. Freeman, "Increased Structure and Active Learning Reduce the Achievement Gap in Introductory Biology," Science (80-. )., vol. 332, no. 6034, pp. 1213-1216, 2011.

[8] T. J. Shaw, S. Yang, T. R. Nash, R. M. Pigg, and J. M. Grim, "Knowing is half the battle: Assessments of both student perception and performance are necessary to successfully evaluate curricular transformation," PLoS One, vol. 14(1): e02, 2019.

[9] M. Stains et al., "Anatomy of STEM teaching in North American universities," Science, vol. 359, no. 6383. 2018. 
[10] N. Nelson and R. Brennan, "Effective Learning Environments: Is there alignment between the ideal, the actual, and the students' perspective?," in CEEA Conference Proceedings 2019, 2019, p. 7.

[11] Faculty Survey of Student Engagement, "FSSE 2018 Aggregate Scales," 2018.

[12] L. S. Shulman, "Those Who Understand: Knowledge Growth in Teaching," Educ. Res., vol. 15, no. 2, pp. 4$14,1986$.

[13] H. L. Dreyfus and S. E. Dreyfus, "Peripheral Vision Expertise in Real World Contexts," Organ. Stud., vol. 26, no. 5, pp. 779-792, 2005.

[14] P. Benner, "From Novice to Expert," Am. J. Nurs., vol. 82, no. 3, pp. 402-407, 1982.

[15] L. J. Lyon, "Development of teaching expertise viewed through the Dreyfus model of skill acquisition," $J$. Scholarsh. Teach. Learn., vol. 15, no. 1, pp. 88-105, 2015.

[16] L. Darling-Hammond, Powerful teacher education: Lessons from exemplary programs. San Francisco, CA: Jossey-Bass, 2006.

[17] J. D. Bransford, A. L. Brown, and R. R. Cocking, How People Learn: Brain, Mind, Experience, and School. Washington, DC: National Academy Press, 2000.

[18] The Royal Academy of Engineering, "Creating cultures where all engineers thrive: A unique study of inclusion across UK engineering," 2017.

[19] L. K. Epting, T. E. Zinn, C. Buskist, and W. Buskist, "Students perspectives on the distinction between ideal and typical teachers," Teach. Psychol., vol. 31, no. 3, pp. 181-183, 2004.

[20] J. Rossetti and P. G. Fox, "Factors Related to Successful Teaching by Outstanding Professors: An Interpretive Study," J. Nurs. Educ., vol. 48, no. 1, 2009.

[21] W. Cerbin, "Improving student learning from lectures.," Scholarsh. Teach. Learn. Psychol., vol. 4, no. 3, pp. 151-163, 2018.

[22] D. Rose, W. Harbour, C. S. Johnston, S. Daley, and L. Abarbanell, Universal Design for Learning in Postsecondary Education, vol. 19, no. 2. 2006.

[23] S. Fowler, Why motivating people doesn't work... and what does: the new science of leading, energizing, and engaging, 1st ed. San Francisco, CA: Berrett-Koehler Publishers, Inc., 2014.

[24] J. B. Ellsworth, Surviving Change - A Survey of Educational Change Models. Syracuse, NY: ERIC Clearinghouse on Information and Technology, 2000.

[25] L. J. Sax, S. K. Gilmartin, and A. N. Bryant, "Assessing Response Rate and Nonreponse bias in Web and Paper Surveys," Res. High. Educ., vol. 44, no. 4, pp. 409-432, 2003. 